\title{
Tutorización virtual sobre matemáticas
}

\author{
Pablo Gregori Huerta, Vicente Martínez García \\ Instituto de Matemáticas y Aplicaciones de Castellón, \\ Departamento de Matemáticas, Universitat Jaume I, \\ Campus del Riu Sec,12071 Castelló de la Plana \\ gregori@uji.es,martinez@uji.es
}

Presentado en eXIDO16 (2016)

\begin{abstract}
RESUMEN
Los profesores de matemáticas, frecuentemente, encuentran problemas en la representación de símbolos para comunicarse de manera virtual con sus estudiantes. En este sentido y con el objetivo de facilitar la comunicación tanto oral como escrita, presentamos diversas opciones realistas de afrontar una tutoría virtual que utilice simbología matemática. Se analiza de forma práctica la comunicación con el uso de ordenador, tableta, webcams y la videoclase según el formato de la Universitat Jaume I de Castellón.
\end{abstract}

Palabras clave: nuevas tecnologías, educación científica, educación virtual.

\section{INTRODUCCIÓN}

Una de las dificultades recurrentes con la que tropiezan los profesores de matemáticas, principalmente, y los profesores de ciencias en general, es la manera de comunicarse a distancia con los estudiantes para resolver las dudas planteadas en clase. Esta dificultad se incrementa cuando nos enfrentamos a la implantación de cursos totalmente online, muy comunes en nuestros días.

La abundancia de la simbología propia de las matemáticas dificulta esta comunicación, el lenguaje escrito no se limita a texto, sino que involucra esquemas, dibujos y símbolos difíciles de representar mediante el teclado habitual del ordenador. La práctica totalidad de alumnos, y un buen número de profesores, no conoce procesadores de texto que ayuden a editar fácilmente preguntas que contengan estos símbolos.

Si la tutoría virtual es asíncrona, el problema tiene una solución más fácil, aunque no exenta de algunos problemas similares cuando ésta es sincrónica. En este caso, se puede usar lápiz y papel, como en el caso clásico presencial, realizar una fotografía con un teléfono móvil o tableta, y adjuntarla a un mensaje de correo electrónico, o bien en un foro o diálogo de Moodle (como ejemplo de plataforma donde se aloja la asignatura en cuestión). 
En el caso complejo, de sincronía, se debe transmitir la explicación en vivo. Para ello, es necesario un dispositivo que capture la señal "analógica" de la escritura y la voz, y la digitalice para ser enviada al otro lado. En este caso, los requisitos tecnológicos para una comunicación fluida, y que se deben dar a ambos lados del canal de comunicación son un ancho de banda de acceso a internet suficiente, y un ordenador de potencia relativa. El requisito adicional para facilitar la escritura matemática sería indispensable al menos para el profesor, pero también para el estudiante si quiere comunicar sus razonamientos por escrito. Este sería un dispositivo táctil con un puntero de precisión, para producir un texto legible.

La preocupación por el uso de herramientas que fomenten un intercambio de comunicación matemática escrita, que emule lo que ocurre en una tutoría presencial clásica de dudas matemáticas, está motivada por la implantación de un grupo a distancia en la docencia del Máster Universitario en Matemática Computacional en la Universitat Jaume I de Castellón (UJI). Una peculiaridad del Máster ---y al mismo tiempo uno de sus puntos fuertes---, es la aceptación del sistema de matrícula no presencial como alternativa al sistema presencial para el alumnado. Así, se pretende orientar al profesorado en la relación con sus alumnos a distancia, estableciendo un método de actuación claro, coordinado y evaluable para, si fuese preciso, realizar aquellas modificaciones que conduzcan a una mejora del aprendizaje a distancia. La implementación de un Modelo Teórico-Práctico de buenas prácticas de movilidad universitaria adaptado al profesorado y estudiantado, no está libre de dificultades. En este sentido, habilitar un procedimiento de comunicación oral y escrita que permita el intercambio de razonamientos entre el profesorado y el estudiantado es clave para el éxito de un correcto aprendizaje del estudiantado a distancia.

\section{COMUNICACIÓN BASADA EN ORDENADOR CON WEBCAM}

El equipamiento básico de un PC portátil o de sobremesa incluye una webcam, micrófono y altavoces, con lo que se hace posible la videoconferencia. Una forma de abordar la comunicación escrita de las matemáticas, que no precise de equipamiento adicional, sería aprovechar la cámara web para enfocar el documento que se desea transmitir por escrito. De este modo, la webcam capturaría la explicación del profesor, y la transmitiría al alumno.

Este tipo de comunicación no es el más eficiente: puede presentar problemas de iluminación, de resolución de la cámara, e incluso de posicionamiento de la webcam. Enfocar una pizarra es relativamente sencillo, pero enfocar un documento sobre la mesa requiere de una webcam externa y un soporte adecuado. Esta opción se incluye porque puede resultar de utilidad a estudiantes con menos recursos, o de países con dificultades en el acceso a Internet de calidad. 
El software más popular, desde hace muchos años, para establecer videollamadas por internet es Skype. Paralelamente, Google ha ido creando un universo de aplicaciones y utilidades web gratuitas, para sus usuarios registrados, entre las que aparecieron las Google Hangouts, que es el equivalente de Skype. Las dos aplicaciones son multiplataforma, proporcionan una ventana de comunicación, y se pueden usar desde cualquier ordenador o dispositivo móvil.

Las características de este tipo de comunicación son:

- La voz se mantiene como elemento esencial en la comunicación entre las personas.

- La webcam, que suele enfocar al interlocutor, se puede:

o Orientar hacia un bloc de notas o pizarra "real", donde el profesor escribe sus explicaciones, y el alumno las ve, al tiempo que oye los comentarios del profesor, y puede aportar sus propios comentarios. De este modo se reproduce la tutoría presencial clásica.

o Conmutar la imagen de la webcam con (parte del) escritorio del ordenador del profesor. De este modo, el profesor puede explicar el funcionamiento de un software científico objeto de estudio, mostrando al alumno los pasos que debe seguir con el software.

\section{COMUNICACIÓN BASADA EN PC Y TABLETA}

Una mejora frente a la situación descrita anteriormente consiste en digitalizar la escritura del profesor con un dispositivo táctil. Esto ayuda a una visualización más clara, e incluso a una gestión eficiente de las notas escritas para su reutilización.

Hace unos años había tabletas muy económicas, pero tenían el inconveniente de ser un soporte sobre el que el puntero no dejaba rastro, de modo que el usuario debía practicar mucho hasta adquirir la destreza de escribir en la tableta, y ver el efecto en la pantalla del ordenador. Pero escribir texto a mano no era operativo. Otras versiones de este tipo de tabletas eran, y son, capaces de reflejar los trazos en su superficie, y se convertían en utilizables. Sin embargo, tienen que competir en precio con las actuales tabletas Android, iOS o Windows, que por un precio muy similar, ofrecen esa captura de escritura y la potencia de un ordenador para ciertas tareas, como la navegación por internet, y la visualización de todo tipo de documentos.

No obstante cabe destacar que no todas las tabletas actuales (de los sistemas operativos comentados) permiten escritura manual de precisión: todas permiten garabatear con la punta del dedo o con un stylus con punta de goma, pero 
para escribir a mano, y más en la habitual simbología matemática, hace falta mayor precisión, y es necesario que la pantalla táctil esté preparada para esa precisión. Desgraciadamente, hasta la fecha, esta característica del producto no se detalla claramente entre las especificaciones técnicas de las tabletas, y hace falta preguntar mucho para saber si están capacitadas.

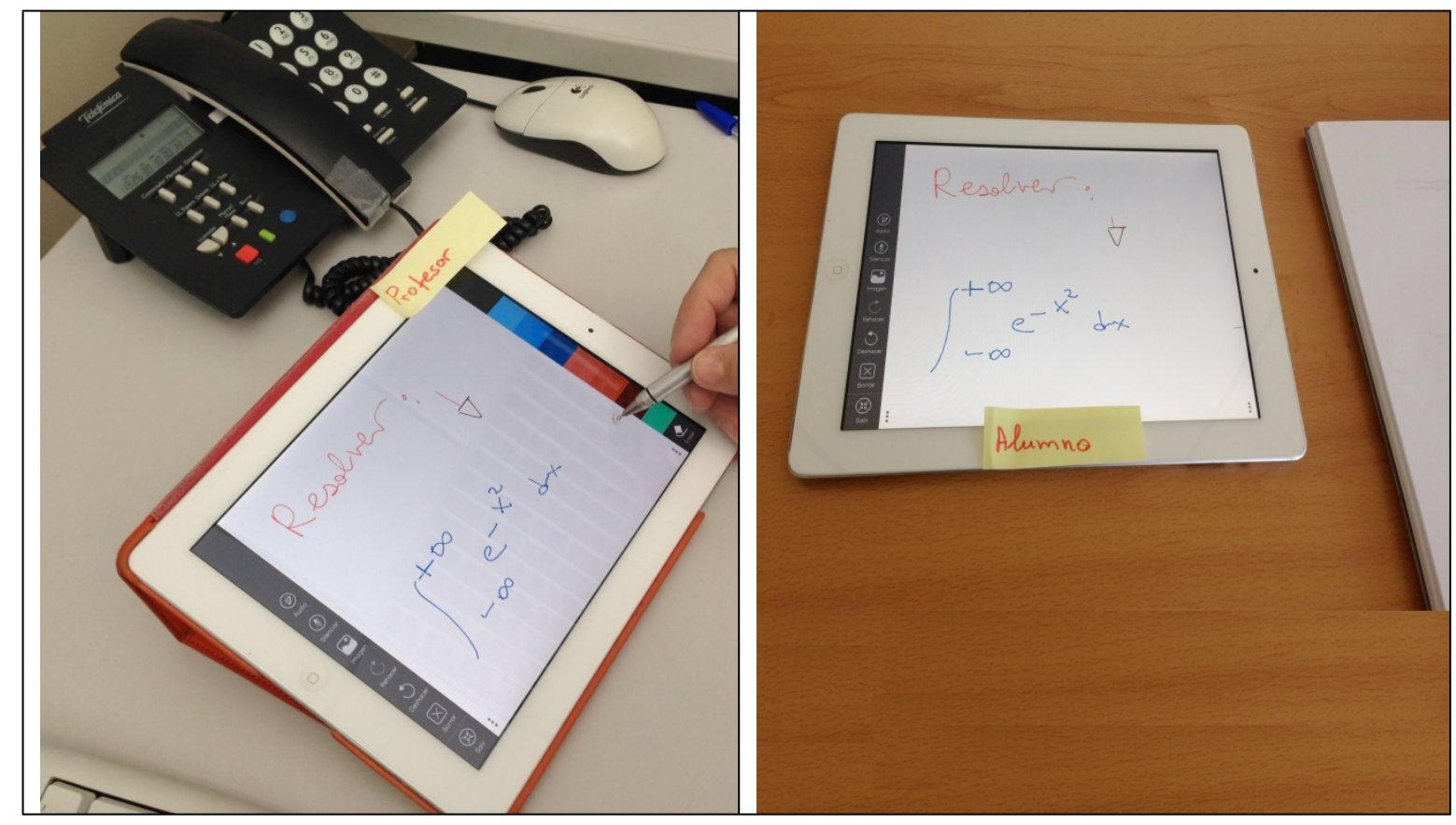

Figura 1. Comunicación mediante tabletas entre profesor y alumno.

Existen tabletas que tienen en su pantalla una doble capacidad sensitiva: una que reconoce el contacto del dedo, y otra que reconoce el contacto de un puntero magnético. Con ellas, la escritura es más cómoda, pudiendo apoyarse la mano al escribir con el puntero, sin que entren en conflicto ambas capacidades. Con una tableta que capte adecuadamente la escritura manual, la comunicación escrita, de profesor a alumno, puede producirse de varias formas:

- TABLETA $\rightarrow$ ORDENADOR $\rightarrow$ VIDECONFERENCIA: el contenido de la pantalla de la tableta pasa a una ventana del escritorio del ordenador del profesor, que se comparte con el alumno durante la videoconferencia.

o TABLETA: El profesor necesita una aplicación para tomar notas manuales, que administre lo que escribe y permita pasar de unas hojas a otras. Ejemplos de aplicación gratuita de ese tipo son: Penultimate (Evernote, 2016) y Squid (Steadfast, 2016). La tableta debe emitir el contenido de su pantalla al ordenador). Los dispositivos de Apple tienen incorporado el protocolo Airplay de 
serie, por lo que no precisan de aplicación. Los dispositivos Android pueden utilizar la aplicación gratuita Mirroring360 Sender Basic (Splashtop, 2016a).

o ORDENADOR: Para recibir la señal de la tableta, necesita un receptor Apple TV, o bien el software Mirroring360 (Splashtop, 2016b), que tiene un coste relativamente económico.

El software de videoconferencia (Skype o Google Hangouts) permite compartir en la conversación, el escritorio completo o cualquiera de sus ventanas, como la que contiene la imagen de la pantalla de la tableta.

- TABLETA $\rightarrow$ NAVEGADOR DE INTERNET: existen páginas web cuyo contenido es un lienzo en blanco sobre el cual los visitantes pueden escribir. Estas webs son operativas cuando se utilizan desde uno de estos dispositivos táctiles. Un ejemplo de ellas es (AWW, 2016).

- TABLETA PROFESOR $\rightarrow$ TABLETA ALUMNO (ver Fig. 1): si profesor y alumno manejan el mismo tipo de dispositivo, pueden interactuar sobre un documento compartido usando una aplicación cualquiera que lo permita, como Talkboard (Citrux, 2016). El usuario crea proyectos que constan de pizarras y colaboradores, y todo queda guardado para futuras sesiones.

\section{VIDEOCLASES EN LA UNIVERSITAT JAUME I DE CASTELLÓN}

Otra herramienta que permite reducir la necesidad de la tutoría es la grabación en vídeo de las clases presenciales y su puesta a disposición, sea de manera privada, dentro de la plataforma (Moodle en el caso de nuestra universidad), en el espacio de la asignatura, o en un sitio público como Youtube.

Una solución local para grabar una clase desde el despacho es usar un programa de grabación del escritorio del ordenador. Dos ejemplos gratuitos comprobados son aTube Catcher (Uscanga, 2011) y CamStudio (Camstudio.org, 2013). Capturan el escritorio (o parte de él) y el sonido que entre por el micrófono. De este modo, usando una tableta, se puede grabar la pizarra en las formas comentadas anteriormente (con una página web de pizarra, o con una aplicación adecuada de la tableta). Para hacer la grabación más amena, y que no se oiga sólo una voz en off, el profesor puede capturar su imagen mediante la webcam del ordenador y, mostrarla en una pequeña ventana situada en una esquina del escritorio, para tapar lo menos posible la pizarra. Dirigiendo su mirada a la webcam de vez en cuando, la grabación de la lección es más personal y agradable para el espectador. 
En el caso de nuestra institución, la mayoría de aulas se han equipado con mesas multimedia, con el hardware y software adecuado, y cámaras de vídeo colocadas en el techo del aula con posiciones preseleccionadas, de modo que el profesor que desea grabar su clase, tan solo tiene que seguir un pequeño número de sencillos pasos:

1) Seleccionar entre:

o Escritorio PC: que tomará la imagen del escritorio.

o Cámara: que tomará la imagen de cámara de vídeo del techo del aula.

o PIP: conmutador que habilita o deshabilita la superposición de la imagen de la cámara del techo, en una esquina del escritorio del ordenador.

2) Caso de elegir "Cámara" o "PIP", el profesor debe seleccionar una de las 4 posiciones establecidas, según el profesor hable (1) desde la silla de la mesa, (2) desde el atril ---que soporta un monitor táctil con puntero de precisión---, (3) desde la pizarra en un plano corto, o (4) desde la pizarra en un plano más amplio.

3) Presionar botón "Grabar" para iniciar la grabación.

4) Presionar botón "Parar" en el momento de finalizar.

Tras detener la grabación, se inicia el procesado del vídeo en un servidor de vídeo, propio de la universidad, de forma que en unos minutos el archivo de vídeo está disponible, de manera privada, para el profesor. Éste puede ingresar en dicho servidor con su cuenta de usuario, y visualizarlo, editarlo o eliminarlo.

Con estas clases, muchas de las tutorías se pueden resolver por el alumno, que tal vez no tuvo tiempo de tomar nota durante la clase de ciertas observaciones del profesor, que después son necesarias para las tareas.

\section{COMENTARIOS FINALES}

Se han presentado diversas opciones realistas de afrontar una tutoría a distancia que utilice simbología matemática, con el objetivo de dar respuesta a uno de los retos planteados en nuestro departamento, consistente en facilitar la comunicación oral y escrita entre el profesorado y alumnado a distancia, propiciando así un correcto aprendizaje.

Resulta evidente que la comunicación más pobre es la primera opción, la cual esta basada en la utilización de un equipo informático básico con webcam. Sin embargo, no hay que despreciarla porque puede haber estudiantes con pocos recursos y ésta ser su única opción.

La utilización de recursos más sofisticados puede facilitar la comunicación y debemos estar atentos a la aparición de nuevas herramientas. El campo de las 
nuevas tecnologías informáticas y de información es muy activo, y las herramientas comentadas hoy, pueden resultar obsoletas en poco tiempo.

Finalmente, agradecemos de antemano a los lectores que quieran compartir con nosotros comentarios y/o nuevas ideas en este campo que, con toda seguridad, nos ayudarán a mejorar nuestro modelo.

\section{REFERENCIAS}

AWW A web whiteboard. (2016). A web whiteboard. Recuperado de https://www.awwapp.com

Camstudio.org. (2013). CamStudio. Recuperado de http://camstudio.org

Citrix. (2016). Talkboard. Recuperado de https://www.talkboard.com

Evernote Corporation. (2016). Penultimate. Recuperado de https://evernote.com/intl/es/penultimate

Steadfast Innovation, LLC. (2016). Squid. Recuperado de http://squidnotes.com

Splashtop. (2016a). Mirroring360 Sender Basic. Recuperado de http://www.mirroring360.com/android

(2016b). Mirroring360. Recuperado de http://www.mirroring360.com

Uscanga, D. (2011). aTube Catcher. Recuperado de http://www.atube.me/video 